\title{
Cardiac Functional and Histopathologic Findings in Humans and Mice with Mucopolysaccharidosis Type I: Implications for Assessment of Therapeutic Interventions in Hurler Syndrome
}

\author{
ELIZABETH BRAUNLIN, SHANNON MACKEY-BOJACK, ANGELA PANOSKALTSIS-MORTARI, JAMES M. BERRY, \\ RON T. MCELMURRY, MEGAN RIDDLE, LI-YAN SUN, LORNE A. CLARKE, JAKUB TOLAR, AND BRUCE R. BLAZAR \\ Department of Pediatrics [E.B., A.P.-M., J.M.B., R.T.M., M.R., L.-Y.S., J.T., B.R.B.], University of Minnesota, Minneapolis, Minnesota \\ 55455; The Jesse E. Edwards Cardiovascular Registry [S.M.-B.], St. Paul, Minnesota 55102; Department of Genetics [L.A.C.], \\ University of British Columbia, Vancouver, BC V5Z 4H4, Canada
}

\begin{abstract}
Hurler syndrome (mucopolysaccharidosis type I [MPS I]) is a uniformly lethal autosomal recessive storage disease caused by absence of the enzyme $\alpha$-L-iduronidase (IDUA), which is involved in lysosomal degradation of sulfated glycosaminoglycans (GAGs). Cardiomyopathy and valvar insufficiency occur as GAGs accumulate in the myocardium, spongiosa of cardiac valves, and myointima of coronary arteries. Here we report the functional, biochemical, and morphologic cardiac findings in the MPS I mouse. We compare the cardiac functional and histopathological findings in the mouse to human MPS I. In MPS I mice, we have noted aortic insufficiency, increased left ventricular size, and decreased ventricular function. Aortic and mitral valves are thickened and the aortic root is dilated. However, murine MPS I is not identical to human MPS I. Myointimal proliferation of epicardial coronary arteries is unique to human MPS I, whereas dilation of aortic root appears unique to murine MPS I. Despite the differences between murine and human MPS I, the murine model provides reliable in vivo outcome parameters, such as thickened and insufficient aortic valves and depressed cardiac function that can be followed to assess the impact of therapeutic interventions in preclinical studies in Hurler syndrome. (Pediatr Res 59: 27-32, 2006)
\end{abstract}

$\mathrm{M}^{\mathrm{s}}$ PS I (Hurler syndrome) is a relatively common (1) lethal lysosomal storage disorder of childhood (2). Absence of the lysosomal hydrolase IDUA results in the accumulation of GAGs heparan sulfate and dermatan sulfate within the CNS, the heart, lungs, liver, bones, and cartilage (2). Excess GAG in MPS I is manifested by corneal clouding, progressive mental retardation, hydrocephalus, deafness, and severe skeletal abnormalities (dysostosis multiplex). Within the human heart, excess GAG is associated with thickened cardiac valves (3), progressive narrowing, and occlusion of the epicardial coronary arteries (4), congestive heart failure, and death in the first decade of life.

Received April 25, 2005; accepted July 27, 2005.

Correspondence: Elizabeth Braunlin, M.D., Ph.D., Department of Pediatrics, University of Minnesota, MMC 94, 420 Delaware St. S.E., Minneapolis, MN 55455; e-mail: braun002@umn.edu

Supported by Minnesota Medical Foundation, Children's Cancer Research Foundation, University of Minnesota Cancer Center, and NIH RO1 HL49997

J.T. and B.R.B. contributed equally to this work.

DOI: 10.1203/01.pdr.0000190579.24054.39
Complete understanding of the phenotypic expression of human MPS I is complicated by a high degree of genetic variability $(5,6)$. Murine models of MPS I, created by the targeted disruption of the gene encoding IDUA in homogeneous murine strains have been advanced as a useful small animal model of this disorder $(7,8)$. Although preliminary cardiac ultrasound and histopathologic data for this model have recently been reported (9), given the potential importance of the murine model in ongoing studies directed toward metabolic correction of MPS I, a more complete understanding of the murine cardiac pathology in this disorder is warranted.

This report is the first to show correlation of functional (echocardiography), biochemical (GAG accumulation) and histopathologic markers of cardiac disease in murine MPS I and to relate these to cardiac disease in human MPS I.

\section{METHODS}

Mice. Adult IDUA gene deletional mutant C57BL/6J mice (MPS I), generated by homologous gene recombination and backcrossed to C57BL/6 more than 12 generations, were obtained as offspring as previously described (7) and bred locally. Reverse-transcriptase polymerase chain reaction was performed on the founders of the C57BL/6J MPS I mouse colony and on random offspring, all showing the expected genomic profile (7). Control C57BL/6J mice were obtained from Jackson Laboratory (Bar Harbor, ME). Mice were housed and handled according to National Institutes of Health and University of Minnesota animal care guidelines. Experimental procedures were approved by University of Minnesota Institutional Animal Care and Use Committee.

GAG content. Myocardium specimens obtained from the heart apex were homogenized using Minibeadbeater-96+ (Biospec Products, Inc., Bartlesville, OK). The homogenate was divided in two parts. One part was tested for GAG content and the other for total protein content. GAGs were extracted by overnight incubation in $0.9 \% \mathrm{NaCl}$ and $0.2 \%$ Triton X-100 (10) and harvested by centrifugation at $8000 \mathrm{rpm}$. GAGs in the supernatant were precipitated with 1,9-dimethylmethylene blue chloride per the manufacturer's instructions (Blyscan Dye Reagent, Accurate Chemical \& Scientific Corporation, Westbury, NY). Precipitate was dissolved in the Blyscan Dissociation Reagent and

Abbreviations: GAG, glycosaminoglycan; IDUA, $\alpha$-L-iduronidase; MPS I, mucopolysaccharidosis type I (Hurler) 
absorbance was measured in triplicate at $650 \mathrm{~nm}$ using a Chameleon 425-100 Multi-label Counter (Hidex, Turku, Finland). Absorbance value was expressed as relative absorbance of chondroitin 4-sulfate standard (Accurate Chemical \& Scientific Corporation). GAG content was normalized to a total protein concentration for each sample tested per the manufacturer's instructions (Dojindo Molecular Technologies, Gaithersburg, MD).

Echocardiography. Due to smaller size, the echocardiographic evaluation in female mice was difficult. Therefore, only male mice were studied. Animals were weighed and their chests were shaved just before study. Adult male MPS I and age- and gender-matched C57BL/6 control mice were lightly anesthetized approximately $15 \mathrm{~min}$ before study with $30 \mu \mathrm{g} / \mathrm{g}$ body weight of intraperitoneal pentobarbital (Abbott Laboratories, North Chicago, IL) dissolved in $50 \mathrm{mg} / \mathrm{mL}$ solution of sterile phosphate-buffered saline. Sedated mice were housed in their cage of origin until studied.

Mice underwent cardiac ultrasound in the supine position under small heating lamps to maintain body temperature. Modified four-chamber, longand short-axis views were obtained using 12- and 15.6-MHz probes and a Sonos 5500 (Philips Medical Systems, Bothell, WA) ultrasound machine. The following parameters were obtained: heart rate, standard M-mode measurements of left ventricular end-diastolic and end-systolic chamber size, left ventricular posterior wall thickness in diastole, Doppler interrogation of mitral and aortic valves. At the completion of study, mice were placed in a heated recovery bay until righting reflex returned and then were returned to their cages.

Murine histopathology. Three 10-mo-old control (C57BL/6) and three 10-mo-old MPS I mice were humanely euthanized and the hearts recovered. Two specimens from each group were cryopreserved in OCT (optimal cutting temperature) medium at $-80^{\circ} \mathrm{C}$ until sectioning. The third specimen from each group was stored in formalin before embedding in paraffin blocks for sectioning. Control and MPS I hearts were positioned in blocks to achieve orientation of the left ventricle and mitral and aortic valves in a view comparable to a long-axis view as defined by cardiac ultrasound (11). Sequential sections from each heart $(5 \mu \mathrm{m})$ were stained for GAG content using toluidine blue and Alcian blue, as well as hematoxylin-eosin and elastin van Gieson (all from Sigma Chemical Co., St. Louis, MO) stains.

Human histopathology. Cardiac specimens from one normal child and two children aged 5 mo and $3 \mathrm{y}$, respectively with documented MPS I, were available for study. Both children with MPS I died suddenly before bone marrow transplantation (BMT). The 5-mo-old child died during preparative regimen for BMT and the 3-y-old child died during a procedure under general anesthesia before conditioning for BMT. Sections from each heart $(5 \mu \mathrm{m})$ were stained with hematoxylin-eosin, elastin van Gieson, and toluidine blue for study.

Data analysis. Differences between measurements of control and MPS I mice were assessed using Fisher's exact test, with $p<0.05$ considered significant.

\section{RESULTS}

GAG accumulation in murine MPS I myocardium increases with age. To determine the degree of GAG accumulation during the lifetime of the MPS I animals, myocardial GAG content was measured in mice aged 3, 7, and 10 mo. The results were compared with GAG accumulation in control mice. There was a statistical trend $(p=0.08)$ toward the accumulation of GAG within the MPS I hearts present by 3 mo of age compared with control mice $(0.28 \pm 0.12$ versus $0.08 \pm 0.06 \mu \mathrm{g}$ of $\mathrm{GAG} / \mathrm{mg}$ of total protein, respectively). Statistically significant differences in GAG content were evident in MPS I versus control mice at 7 mo of age $(0.78 \pm 0.22$ versus $0.07 \pm 0.02 \mu \mathrm{g}$ of $\mathrm{GAG} / \mathrm{mg}$ of total protein; $p=0.03$ ) and at $10 \mathrm{mo}$ of age $(1.59 \pm 0.35$ versus $0.06 \pm 0.04 \mu \mathrm{g}$ of $\mathrm{GAG} / \mathrm{mg}$ of total protein; $p=0.0015$, Fig. 1).

Cardiac valves are thickened and elastin fiber disarray is present in both murine and human MPS I. Histopathologically, the aortic valves of both human (Fig. $2 A$ and $B$ ) and mouse (Fig. $2 C$ and $D$ ) with MPS I were markedly thickened and abnormal (Fig. $2 B$ and $D$ ) when compared with their respective normals (Fig. $2 A$ and $C$ ). The MPS I aortic valves demonstrated expansion of the spongiosa and fibrosa layers by

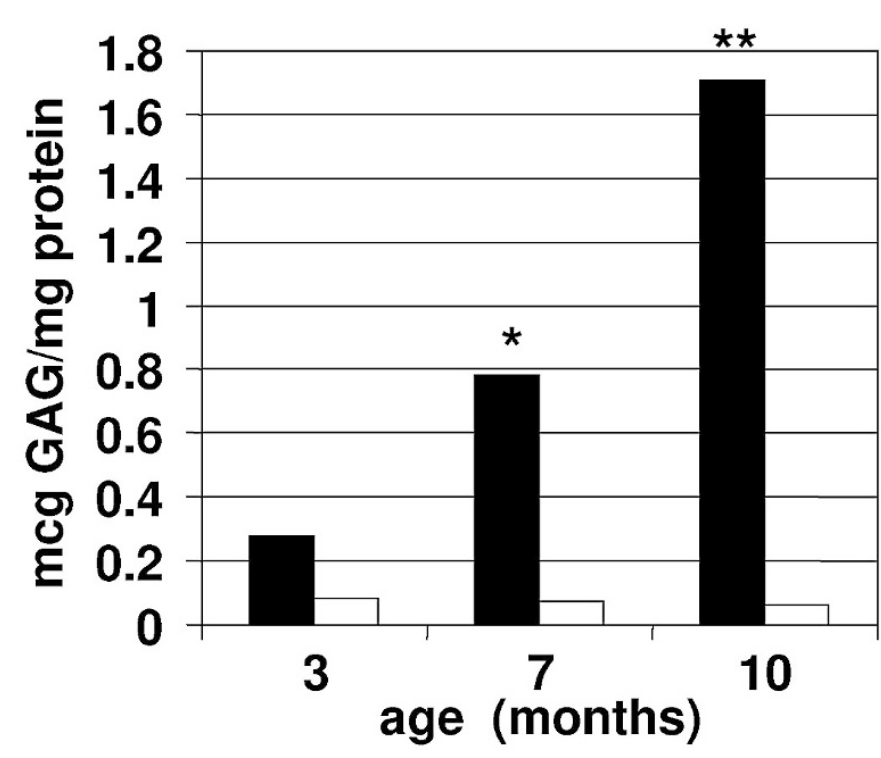

Figure 1. GAG content within myocardium of MPS I mice increases with age. GAG content in myocardium of MPS I mice ( $\mathbf{\square})$ is statistically greater than C57BL/6 Control mice $(\square)$ at $7(* p=0.03)$ and $10(* * p=0.0015) \mathrm{m}$ of age. Values shown represent average of three or four animals per group, normalized to total protein.
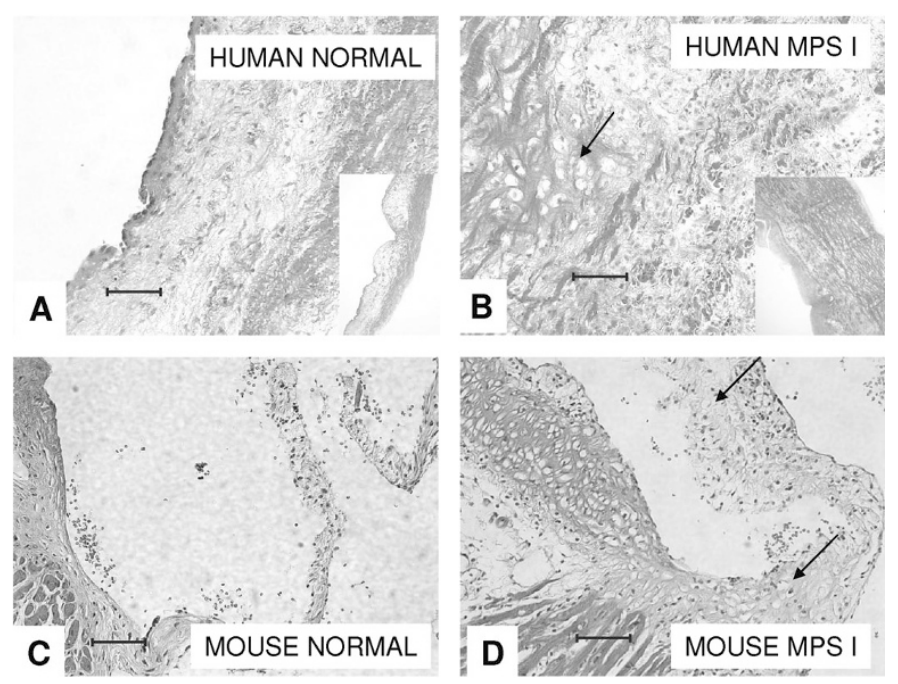

Figure 2. Thickened aortic valves in MPS I. Hematoxylin-eosin stain from a normal child $(\times 200$; insert, $\times 40)(A)$, MPS I human, $(\times 200$; insert, $\times 40)(B)$, control mouse $(\times 200)(C)$, MPS I mouse $(\times 200)(D)$. Valve thickening and cellular deposition of GAG within vacuolated clear (valvular interstitial) cells are seen in both human $(B)$ and murine $(D)$ MPS I (arrows). Scale bar $(\times 200)$ $=100 \mu \mathrm{m}$

clear cells containing GAG (Fig. $2 B$ and $D$ ) and loosely arrayed collagen. When using an elastin stain, a paucity and disarray of elastin fibers were identified within both human and murine MPS I valves (Fig. $3 B$ and $D$ ) and clear cells were easily demonstrable (Fig. $2 B$ and $D$ ). Alcian blue staining in the valve leaflets of the MPS I mouse (Fig. $4 A$ and $B$ ) identified increased GAG content within the valve. Effacement of the sinotubular ridge and dilation of the aortic root (Fig. $4 A$ and $B$ ) was also noted in the MPS I mouse.

The mitral and tricuspid valves (Fig. $5 A-D$ ) of the MPS I humans (Fig. 5B) and MPS I mice (Fig. 5D) appeared thick- 

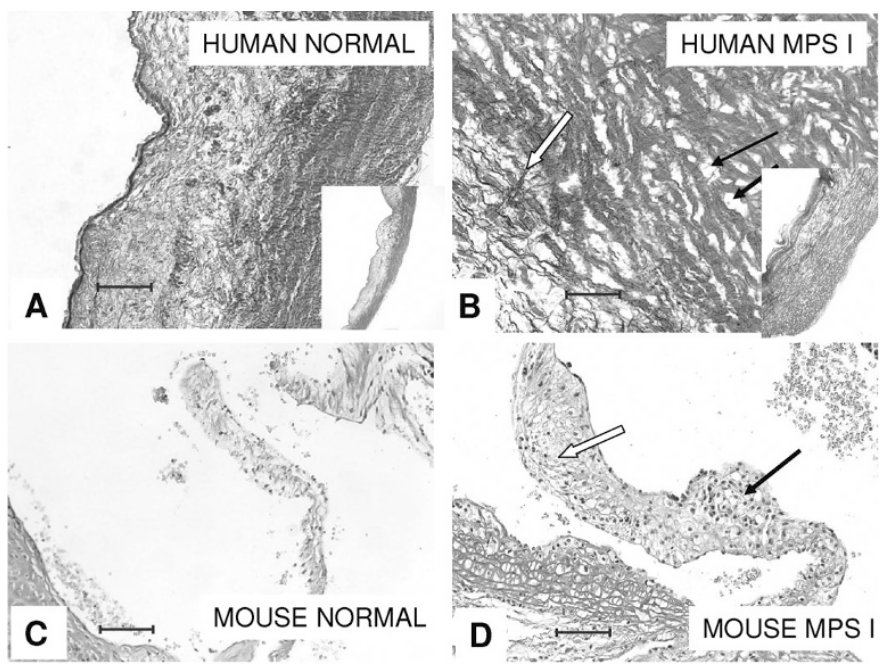

Figure 3. Paucity and disarray of elastin fibers in MPS I aortic valves. Elastin Van Gieson stain from a normal child $(\times 200$; insert, $\times 40)(A)$, a human with MPS I $(\times 200$; insert, $\times 40)(B)$, control mouse $(\times 200)(C)$, MPS I mouse $(\times 200)(D)$. Note markedly thickened aortic valve leaflets $(B$ and $D)$ of both human $(B$, insert) and murine MPS I aortic valves. Multiple areas of intracellular GAG deposition (black arrows) and paucity of elastin with disarray (white arrows) are present in both human and mouse MPS I aortic valves. Scale bar $\times 200=100 \mu \mathrm{m}$.

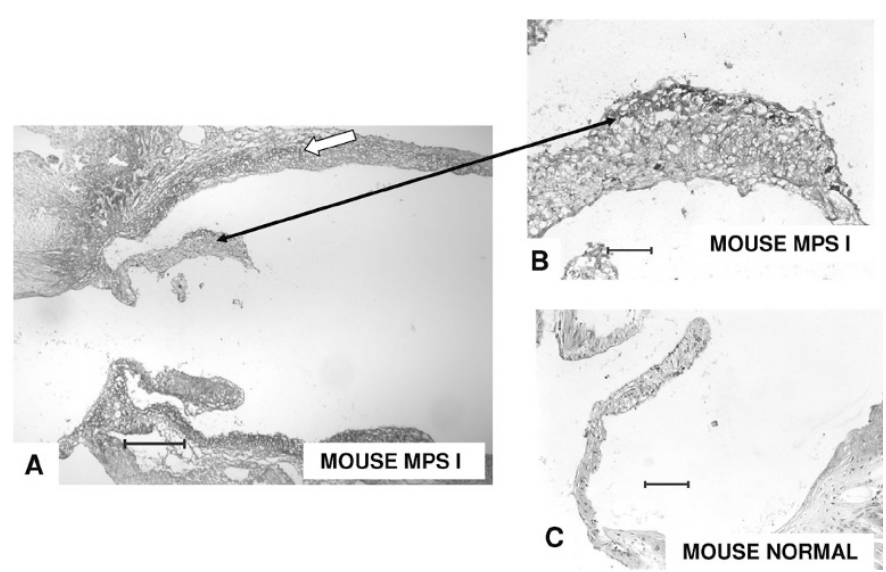

Figure 4. GAG deposition in MPS I aortic valve. Alcian blue stain from MPS I mouse aortic root $(\times 40)(A)$, MPS I mouse aortic valve $(\times 200)(B)$, and control mouse aortic valve $(\times 200)(C)$. Note markedly dilated aortic root (white arrow) and intracellular deposition of Alcian blue positive material (black arrow) within MPS I valve, consistent with increased GAG content. Scale bars: $\times 40=500 \mu \mathrm{m} ; \times 200=100 \mu \mathrm{m}$.

ened, though to a lesser degree than aortic valves when compared with their respective normals (Fig. $5 A$ and $C$ ). Expansion of the valve spongiosa by clear cells (Fig. $5 B$ and $D$ ) and by amorphous collagen accounted for the increase in valve thickness.

Aortic root dilatation occurs in murine but not human MPS I. The aortic walls of both human (Fig. 6B) and mouse (Fig. 6D) with MPS I were markedly thickened by the presence of GAG containing cells deposited between elastin fibers when compared with their respective normals (Fig. $6 A$ and $C$ ). A paucity of elastin fibers and disruption of these fibers were identified in both human and murine MPS I, but not in normals. Increased aortic diameter and effacement of aortic sinuses were present in the murine expression of MPS I (Fig.
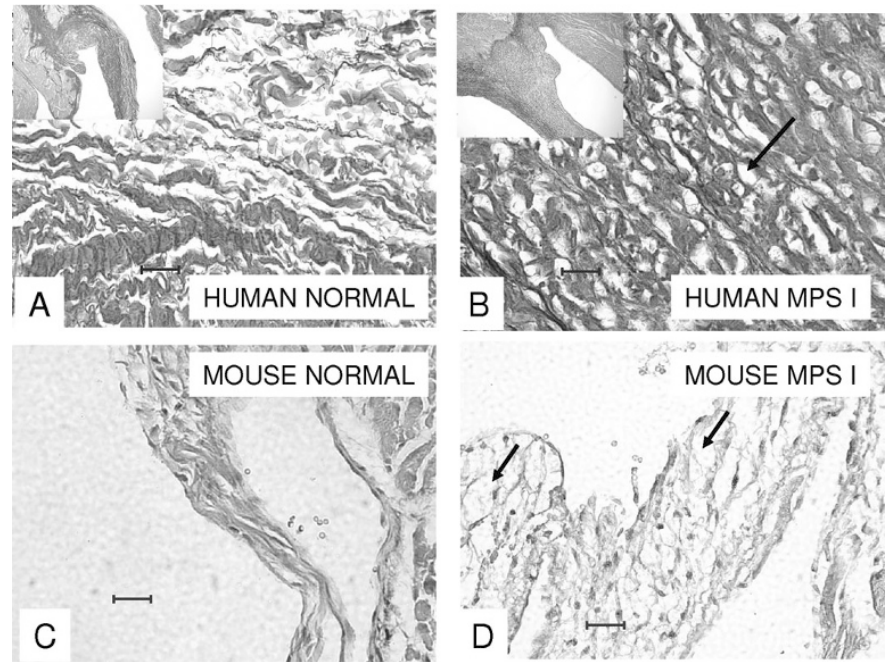

Figure 5. Thickened atrioventricular valves in MPS I. Elastin van Gieson stains from normal child's tricuspid valve $(\times 400$; insert, $\times 40)(A)$, MPS I human mitral valve $(\times 400$; insert, $\times 40)(B)$, control mouse mitral valve $(\times 400)(C)$, and MPS I mouse mitral valve $(\times 400)(D)$. Note expansion of atrioventricular valves in both human and mouse MPS I by deposition of GAG within vacuolated (valvular interstitial) cells (arrows). Scale bar $\times 400$ $=50 \mu \mathrm{m}$.
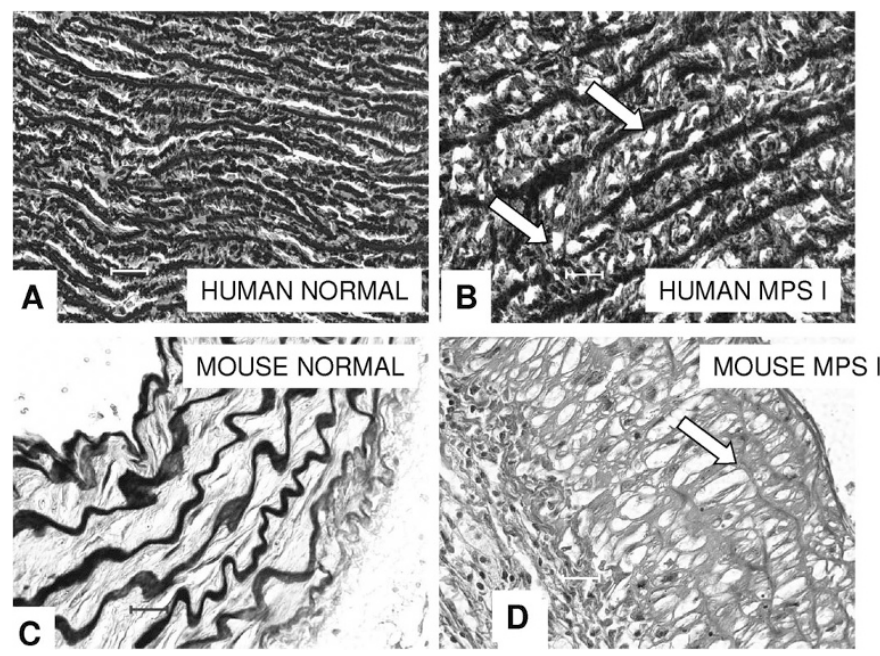

Figure 6. Thickened aortic walls in MPS I. Elastin van Gieson stain from a normal child $(\times 400)(A)$, human with MPS I $(\times 400)(B)$, control mouse $(\times 400)(C)$, and MPS I mouse $(\times 400)(D)$. Note relative paucity of elastin fibers and expansion by GAG deposition within vacuolated (valvular interstitial) cells (white arrows) in both human and murine expressions of MPS I. Scale bar $\times 400=50 \mu \mathrm{m}$.

$4 A$ and Fig. $7 C$ ), but not in the human MPS I specimens studied (Fig. 7A).

Myointimal proliferation does not occur in murine MPS I epicardial coronary arteries. The epicardial coronary arterial lumens of humans with MPS I (Fig. $8 C$ and $D$ ) were dramatically narrowed by the presence of myointimal proliferation by collagen and clear cells (versus controls (Fig. $8 A$ and $B$ ). In contrast, the epicardial coronary arteries of the MPS I mouse appeared dilated, myointimal proliferation was noticeably absent, and the elastin tissue within the arterial walls appeared fragmented and sparse (Fig. $7 B$ and $D$ ).

On light microscopy, there were few differences between the human and murine hearts with MPS I (data not shown). 


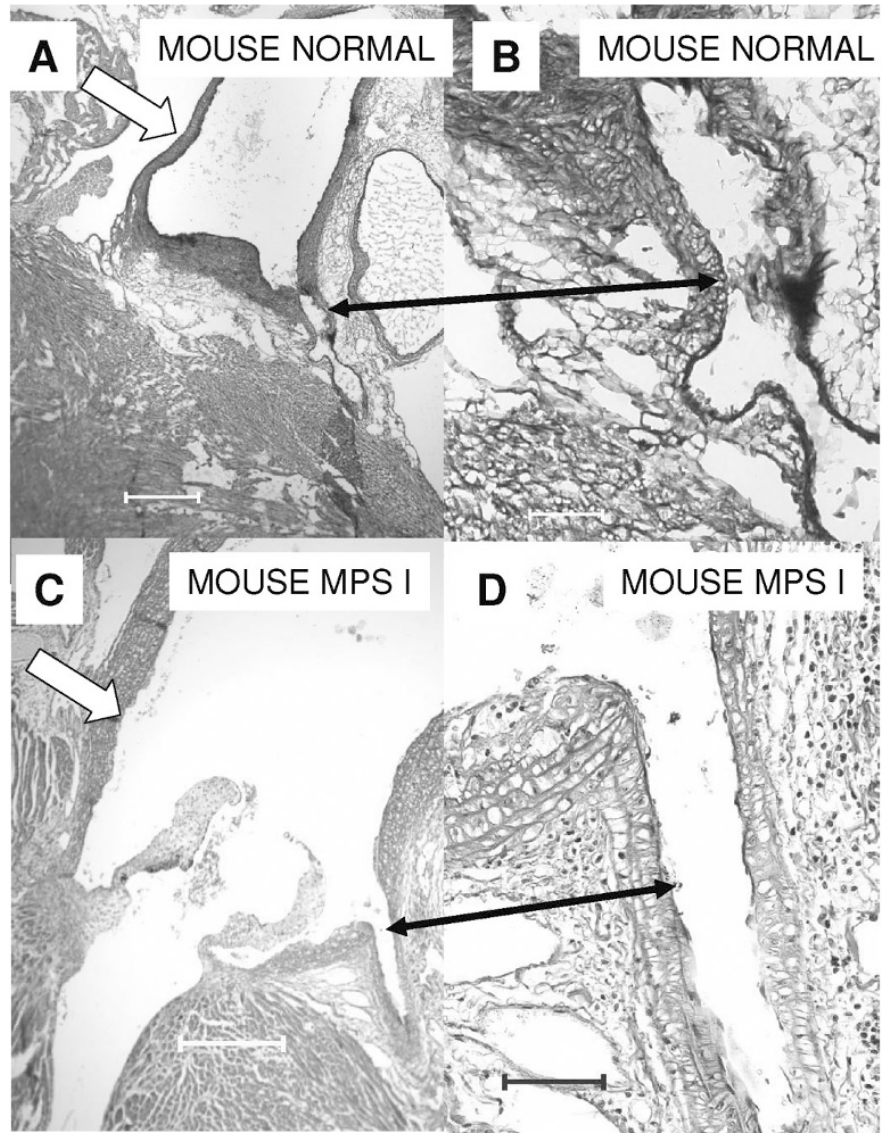

Figure 7. Aortic root and origin of epicardial coronary artery in mice. Elastin van Gieson stain from control mouse $(\times 40)(A)$, control mouse $(\times 200)(B)$, MPS I mouse $(\times 40)(C)$, and MPS I mouse $(\times 200)(D)$. Note dilation of aorta and effacement of aortic sinuses in MPS I mouse $(C)$ compared with control (A) (white arrows). Aortic walls are thickened in MPS I mouse and elastin is decreased. Epicardial coronary arteries are widely patent in both control $(B)$ and MPS I $(D)$ mice (arrows). Scale bars: $\times 40=500 \mu \mathrm{m}, \times 200=100 \mu \mathrm{m}$.

Myocytes appeared well preserved in the MPS I hearts, and there was scant evidence of increased GAG deposition. Intramyocardial coronary arteries were widely patent in both human and murine MPS I hearts.

MPS I mice have abnormal cardiac ultrasound findings. MPS I mice had significantly increased left ventricular enddiastolic dimension $(p=0.001)$ and decreased shortening fraction $(p=0.005)$ when compared with controls (Table 1$)$. Aortic insufficiency was present in 10 of 10 MPS I mice and 0 of 10 age-matched controls $(p=0.0001$, Fig. 9). No significant difference was found between MPS I and control mice in left ventricular posterior wall thickness $(p=0.48$, Table 1).

In mice, pentobarbital can depress heart rate and heart contractility (12). There was no significant difference in heart rate between MPS I and control mice (MPS I, $n=9,517$ beats/min versus control, $n=10,471.6$ beats $/ \mathrm{min} ; p=0.57$ ). To demonstrate that decreased function is not an effect of sedation, shortening fraction was plotted against heart rate (Fig. 10). Shortening fraction of the MPS I mice was depressed regardless of heart rate when compared with controls.

There were no significant differences in age or weight between MPS I and controls (Table 1). MPS I versus control

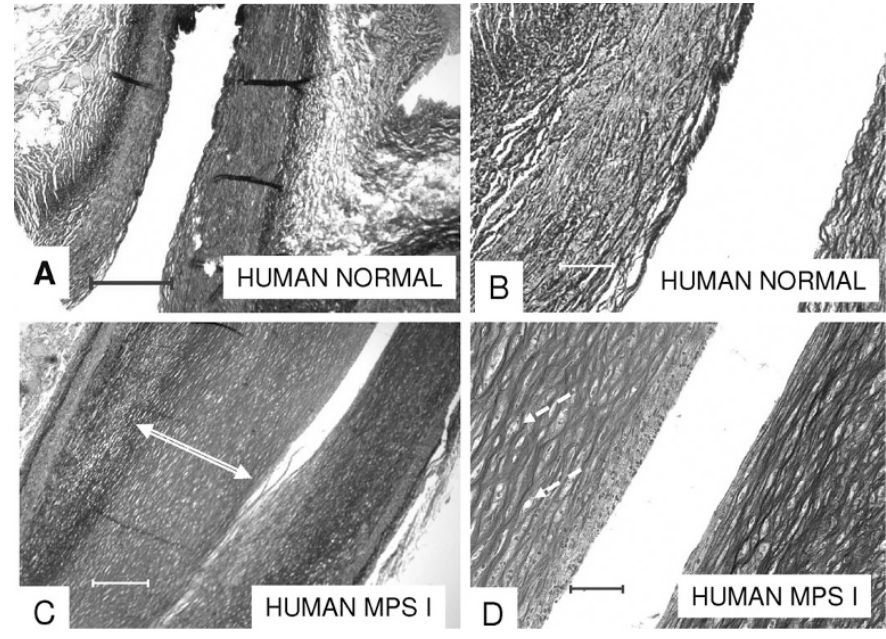

Figure 8. Epicardial coronary arteries in humans. Elastin van Gieson stains from a normal child $(\times 40)(A)$ and $\times 200(B)$ and a human with MPS I $(\times 40)$ $(C)$ and $\times 200(D)$. Contrast the pronounced myointimal proliferation (long arrow) and nearly occluded human MPS I epicardial coronary artery $(C)$ with widely patent normal epicardial coronary artery $(A$ and $B)$. Deposition of GAG is present within the myointima of MPS human coronary arterial walls (short arrows) and elastin fibers appear sparse when compared with the normal human. Scale bars: $\times 40=500 \mu \mathrm{m}, \times 200=100 \mu \mathrm{m}$.

mice were approximately 8 mo of age at the time of study $(249.9 \pm 42.3 \mathrm{~d}$ versus $240.7 \pm 28.0 \mathrm{~d}, p=0.57)$. Average weight of MPS I mice was $33.2 \pm 2.0 \mathrm{~g}$ versus $33.2 \pm 3.0 \mathrm{~g}$ for controls $(p=0.99)$.

\section{DISCUSSION}

The purpose of this study was to define the cardiac functional and histopathologic similarities and differences between the human and murine expressions of MPS I. The mouse model has been used as a surrogate in approaches directed toward metabolic correction of MPS I using retrovirally transduced bone marrow and gene therapy with direct injection of lentiviral, retroviral, adeno-associated virus vectors $(9,10,13,14)$. Detailed functional and morphologic studies of the murine MPS I heart, however, have not been reported. This information is important since correction of MPS I in humans (15) by BMT has yielded mixed results with respect to the heart (16-18). Although late death from coronary artery occlusion has not been reported in humans after BMT, deposition of GAG appears to progress unabated in human cardiac valves (18). Identifying and correcting functional and morphologic abnormalities in the MPS I mouse heart could ultimately have implications for the application of novel therapies in the human MPS I disease.

The most obvious histopathologic similarities between murine and human MPS I hearts are thickening of the cardiac valves and great vessels from cellular deposition of GAGs and collagen (Table 2). Other noticeable similarities between the two models include absence of myointimal proliferation within the intramyocardial coronary arteries and the benign histopathologic appearance of the myocardium by light microscopy, even though murine myocardial GAG content clearly increases with age (Fig. 1). In spite of these similari- 
Table 1. Echocardiographic findings of control and MPS I mice

\begin{tabular}{lccccccr}
\hline & Age $(\mathrm{d})$ & Weight $(\mathrm{g})$ & LVPW $(\mathrm{cm})$ & LVEDD $(\mathrm{cm})$ & LV mass $(\mathrm{g})$ & SF & Aortic insufficiency \\
\hline MPS I $(n=10)$ & $249.9 \pm 42.3$ & $33.2 \pm 2.0$ & $0.10 \pm 0.01$ & $0.53 \pm 0.15$ & $0.83 \pm 0.13$ & $0.29 \pm 0.11$ \\
Control $(n=10)$ & $240.7 \pm 28.0$ & $33.2 \pm 3.0$ & $0.11 \pm 0.01$ & $0.35 \pm 0.04$ & $0.71 \pm 0.01$ & $0.44 \pm 0.10$ & $10 / 10$ \\
$p$ value & 0.57 & 0.99 & 0.48 & 0.001 & 0.009 & 0.005 & 0.10 \\
\hline
\end{tabular}

LVPW, left ventricular posterior wall; LVEDD, left ventricular end-diastolic dimension; LV mass, left ventricular mass; SF, shortening fraction.

ties, there are physiologic and histopathologic differences between the two models.

By cardiac ultrasound, aortic insufficiency appears unique to the murine model (Table 2), while the mitral valve remains competent. Additional ultrasound evaluation of aortic and mitral valves by high-resolution ultrasound biomicroscope (VisualSonics, Toronto, Ontario) has confirmed these results. By contrast, aortic insufficiency is uncommon in human MPS I and mitral abnormalities predominate (18-20). Left ventricular end-diastolic dimension is increased in both human (18) and murine MPS I. Depressed cardiac function is not a constant feature of human MPS I (18-20) but is reliably present in our mice.

Histologically, progressive myointimal proliferation within the epicardial coronary arteries is absent in the MPS I mouse (Table 2) and invariably present in human MPS I (4). It is relevant to cardiac pathology studies that the spontaneous development of coronary atherosclerosis in inbred mice is unknown (21). It is known that both heparin sulfate and dermatan sulfate are more prominent within the murine vasculature than the human $(22,23)$; thus, the absence of myointimal proliferation within the murine MPS I coronaries is even more remarkable.

Aortic root dilation and effacement of aortic sinuses seen here in MPS I mice, have not been reported in MPS I humans (Table 2). This histopathologic picture is similar to that seen in human Marfan syndrome (24). The transgenic mouse model of Marfan syndrome homozygous for mutant fibrillin-1 (25) develops a similar aneurysmal dilation of the proximal aorta but, in contrast to the MPS I mouse, succumbs at an early age. Histologically, the aortic wall is thinned in the fibrillin-1 mutant, not thickened by GAG containing cells, as is seen in the MPS I mouse.

Accumulation of the GAG dermatan sulfate inhibits elastin fiber assembly in human MPS I hearts (26). This inhibition of elastin assembly results in a paucity of elastin fibers and

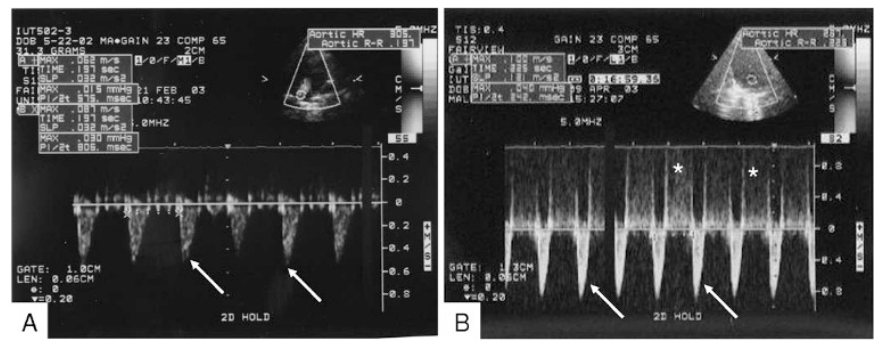

Figure 9. Aortic insufficiency develops in MPS I mice. Doppler signal from aortic valve of a control mouse $(A)$ and MPS I mouse $(B)$. Downward flow velocity signals (arrows) from both control and MPS I mice represent antegrade aortic flow. Regurgitant flow across the aortic valve is identified (asterisk) in MPS I mice only. regurgitant cardiac valves, but dilation of the aortic root in humans has not been reported. In the MPS I mouse heart, paucity of elastin appears most pronounced in the aortic walls (Fig. 6D). Dilation of the aortic root and effacement of the aortic sinuses is seen both by ultrasound as well as by histopathology and may be responsible in part for regurgitation of the aortic valve in MPS I mice.

Species differences in IDUA gene mutation may explain some of the histopathologic differences seen in humans and mice with MPS I. The two murine MPS I models described to date $(7,8)$ are similar to each other, as they have been generated in the same murine strain $(\mathrm{C} 57 \mathrm{BL} / 6)$ by insertion of neomycin resistance gene in the same exon of the IDUA gene (exon 6). The genetic diversity of human MPS I, however, far exceeds that of the mouse model, since more than 50 pathologic mutations of the IDUA gene have been characterized from patients with MPS I $(5,6)$, and it is well recognized that the genotype-phenotype correlation is far from complete (5).

The cardiac ultrasound markers that we describe in the MPS I mouse, while not identical to those of the human disease, are critical in assessing the response of cardiac pathology in MPS I to therapeutic interventions, such as stem cell transplantation, enzyme replacement, or gene therapy. Alleviation of aortic valve regurgitation in this model would demonstrate normalization of tissue sites in the heart currently inaccessible to the beneficial effects of BMT (18). It is possible and consistent with our findings that MPS I mice develop valvar disease at younger age (relative to human MPS I patients). The

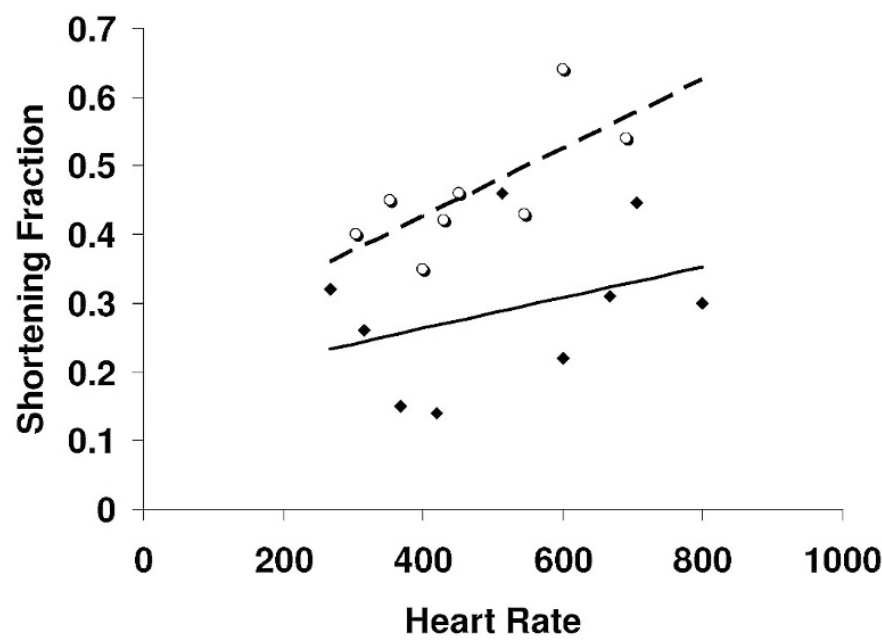

Figure 10. Myocardial function is lower for MPS I mice at all heart rates. There was no significant difference in heart rate between MPS I and control mice (MPS I, $n=9 ; 517$ beats/min vs control, $n=10 ; 471.6$ beats $/ \mathrm{min} ; p=$ 0.57). When compared with control mice ( $\bigcirc$; dashed line) depression of myocardial function at comparable heart rates is seen in MPS I ( $\bullet$ solid line). 
Table 2. Comparison of human and murine cardiac findings

\begin{tabular}{lll}
\hline \multicolumn{1}{c}{ Parameter } & \multicolumn{1}{c}{ Human (ref.) } & Murine (ref.) \\
\hline Echocardiography & & Increased (present study) \\
Left ventricular size & Increased (18,20) & Decreased (present study) \\
Left ventricular contractility & Normal (18); normal or decreased (19,20) & Common (9, present study) \\
Aortic insufficiency & Uncommon (18-20) & Absent (9, present study) \\
Mitral insufficiency & Common (18-20) & Common (9, present study) \\
Histopathology & Common (3) & Common (9, present study) \\
Valves: GAG deposition & Common (26) & Common (9, present study) \\
Valves: decreased elastin & Unreported & Common (9, present study) \\
Aortic root dilation & Common (3) & Common (9, present study) \\
Aortic root: GAG deposition & Unreported & Normal (present study) \\
Aortic root: decreased elastin & Myointimal proliferation (4) & Normal (present study) \\
Epicardial coronary arteries & Normal (4) & \\
Intramyocardial coronary arteries & &
\end{tabular}

mouse model, therefore, may represent a more aggressive form of MPS I, which makes it more beneficial for preclinical assessment of stem cell- or enzyme or gene transfer-mediated therapeutic interventions.

In summary, the MPS I mouse model is not identical to human MPS I but displays reliable in vivo markers, namely, thickened and insufficient aortic valves, aortic dilation, depressed cardiac function, and left ventricular enlargement. As a functional correlate of GAG accumulation seen in both human and murine MPS I, these in vivo parameters can be followed by cardiac ultrasound to assess response in preclinical models of therapeutic intervention for cardiac disease in Hurler syndrome.

\section{REFERENCES}

1. Meikle PJ, Hopwood JJ, Clague AE, Carey WF 1999 Prevalence of lysosomal storage disorders. JAMA 281:249-254

2. Neufeld EF, Muenzer J. 1997 The mucopolysaccharidoses. In: Scriver CR, Beaudet AL, Sly WS, Valle D (eds) The Metabolic Basis of Inherited Diseases. McGrawHill, New York, pp 2465-2494

3. Renteria VG, Ferrans VJ, Roberts WC 1976 The heart in the Hurler syndrome: gross, histologic and ultrastructural observations in five necropsy cases. Am J Cardiol 38:487-501

4. Brosius FC, 3rd, Roberts WC 1981 Coronary artery disease in the Hurler syndrome. Qualitative and quantitative analysis of the extent of coronary narrowing at necropsy in six children. Am J Cardiol 47:649-653

5. Beesley CE, Meaney CA, Greenland G, Adams V, Vellodi A, Young EP, Winchester BG 2001 Mutational analysis of 85 mucopolysaccharidosis type I families: frequency of known mutations, identification of 17 novel mutations and in vitro expression of missense mutations. Hum Genet 109:503-511

6. Matte U, Yogalingam G, Brooks D, Leistner S, Schwartz I, Lima L, Norato DY, Brum JM, Beesley C, Winchester B, Giugliani R, Hopwood JJ 2003 Identification and characterization of 13 new mutations in mucopolysaccharidosis type I patients. Mol Genet Metab 78:37-43

7. Clarke LA, Russell CS, Pownall S, Warrington CL, Borowski A, Dimmick JE, Toone J, Jirik FR 1997 Murine mucopolysaccharidosis type I: targeted disruption of the murine $\alpha$-L-iduronidase gene. Hum Mol Genet 6:503-511

8. Ohmi K, Greenberg DS, Rajavel KS, Ryazantsev S, Li HH, Neufeld EF 2003 Activated microglia in cortex of mouse models of mucopolysaccharidosis I and IIIB. Proc Natl Acad Sci U S A 100:1902-1097

9. Liu Y, Xu L, Hennig AK, Kovacs A, Fu A, Chung S, Lee D, Wang B, Herati RS, Mosinger Ogilvie J, Cai SR, Parker Ponder K 2005 Liver-directed neonatal gene therapy prevents cardiac, bone, ear and eye disease in mucopolysaccharidosis I mice. Mol Ther 11:35-47

10. Zheng Y, Rozengurt N, Ryazantsev S, Kohn DB, Satake N, Neufeld EF 2003 Treatment of the mouse model of mucopolysaccharidosis I with retrovirally transduced bone marrow. Mol Genet Metab 79:233-244

11. Snider AR, Serwer GA 1990 Echocardiography in Pediatric Heart Disease. Year Book Medical Publishers, Chicago, p 23

12. Yang XP, Liu YH, Rhaleb NE, Kurihara N, Kim HE, Carretero OA 1999 Echocardiographic assessment of cardiac function in conscious and anesthetized mice. Am J Physiol 277:H1967-H1974

13. Di Domenico C, Villani GR, Di Napoli D, Reyero EG, Lombardo A, Naldini L, Di Natale P 2005 Gene therapy for a mucopolysaccharidosis type I murine model with lentiviral-IDUA vector. Hum Genet Ther 16:81-90

14. Hartung SD, Frandsen JL, Pan D, Koniar BL, Graupman P, Gunther R, Low WC, Whitley CB, McIvor RS 2004 Correction of metabolic, craniofacial, and neurologic abnormalities in MPS I mice treated at birth with adeno-associated virus vector transducing the human $\alpha$-L-iduronidase gene. Mol Ther 9:866-875

15. Hobbs JR, Hugh-Jones K, Barrett AJ, Byrom N, Chambers D, Henry K, James DC, Lucas CF, Rogers TR, Benson PF, Tansley LR, Patrick AD, Mossman J, Young EP 2004 Reversal of clinical features of Hurler's disease and biochemical improvement after treatment by bone-marrow transplantation. Lancet 2:709-712

16. Vinallonga X, Sanz N, Balaguer A, Miro L, Ortega JJ, Casaldaliga J 1992 Hypertrophic cardiomyopathy in mucopolysaccharidosis: regression after bone marrow transplantation. Pediatr Cardiol 13:107-109

17. Gatzoulis MA, Vellodi A, Redington AN 1995 Cardiac involvement in mucopolysaccharidosis: effects of allogenic bone marrow transplantation. Arch Dis Child 73:259-260

18. Braunlin EA, Stauffer NR, Peters CH, Bass JL, Berry JM, Hopwood JJ, Krivit W 2003 Usefulness of bone marrow transplantation in the Hurler syndrome. Am J Cardiol 92:882-886

19. Dangel JH 1998 Cardiovascular changes in children with mucopolysaccharide storage diseases and related disorders - Clinical and echocardiographic findings in 64 patients. Eur J Pediatr 157:534-538

20. Gross DM, Williams JC, Caprioli C, Dominguez B, Howell RR 1988 Echocardiographic abnormalities in the mucopolysaccharide storage diseases. Am J Cardiol. 61:170-176

21. Daugherty A 2002 Mouse models of atherosclerosis. Am J Med Sci 323:3-10

22. Stefanovich V, Akiyama K 1970 Comparative studies of aortic acid mucopolysaccharides in fifteen species. Comp Biochem Physiol 34:125-130

23. Gardais A, Picard J, Hermelin B 1973 Glycosaminoglycan (GAG) distribution in aortic wall from five species. Comp Biochem Physiol B 44:507-515

24. Brown OR, DeMots H, Kloster FE, Roberts A, Menashe VD, Beals RK 1975 Aortic root dilatation and mitral valve prolapse in Marfan's syndrome: an echocardiographic study. Circulation 52:651-657

25. Pereira L, Lee SY, Gayraud B, Andrikopoulos K, Shapiro SD, Bunton T, Biery NJ, Dietz HC, Sakai LY, Ramirez F 1999 Pathogenetic sequence for aneurysm revealed in mice underexpressing fibrillin-1. Proc Natl Acad Sci U S A 96:3819-3823

26. Hinek A, Wilson SE 2000 Impaired elastogenesis in Hurler disease. dermatan sulfate accumulation linked to deficiency in elastin-binding protein and elastic fiber assembly. Am J Pathol 156:925-938 